\title{
Miranda
}

Revue pluridisciplinaire du monde anglophone /

Multidisciplinary peer-reviewed journal on the English-

speaking world

$20 \mid 2020$

Staging American Nights

« Find your way from darkness to light »: la nuit et l'héritage de la tradition romantique dans Knight of Cups de Terrence Malick

\section{Guilain Chaussard}

\section{OpenEdition}

Journals

Édition électronique

URL : http://journals.openedition.org/miranda/25071

DOI : 10.4000/miranda.25071

ISSN : 2108-6559

Éditeur

Université Toulouse - Jean Jaurès

Référence électronique

Guilain Chaussard, « «Find your way from darkness to light » : la nuit et l'héritage de la tradition romantique dans Knight of Cups de Terrence Malick », Miranda [En ligne], 20 | 2020, mis en ligne le 04 mai 2020, consulté le 16 février 2021. URL : http://journals.openedition.org/miranda/25071 ; DOI : https://doi.org/10.4000/miranda.25071

Ce document a été généré automatiquement le 16 février 2021.

\section{cc)}

Miranda is licensed under a Creative Commons Attribution-NonCommercial-NoDerivatives 4.0

International License. 


\title{
« Find your way from darkness to light » : la nuit et l'héritage de la tradition romantique dans Knight of Cups de Terrence Malick
}

\author{
Guilain Chaussard
}

\section{Introduction}

1 Knight of Cups est le second volet d'une trilogie au cours de laquelle Malick en est venu à rompre avec son sujet de prédilection - les grands espaces et l'Amérique du passé pour porter à l'écran une réflexion sur la vie moderne et sur l'amour ${ }^{1}$. Placé sous le signe du Voyage du Pèlerin (The Pilgrim's Progress, 1678) de John Bunyan, le film se présente comme un récit initiatique. De façon typique, Malick fait apparaître son protagoniste sur une plage, pointant du doigt le soleil de l'Ouest, tandis qu'une voix dit : « Find your way from darkness to light ».

2 Si dans le cadre de ce récit symbolique, la «nuit» survient en une demi-douzaine d'occasions, ses interventions ne durent chaque fois que quelques minutes et sa récurrence peut facilement passer inaperçue à la première vision. Un postulat est que son étude permet pourtant de mettre au jour la richesse esthétique du film et sa signification. On envisagera que la «nuit» y occupe une place analogue à celle d'un motif au sein d'une composition musicale. À cette fin, l'on reprendra la définition d'Emmanuelle André selon laquelle le motif est « l'un des lieux où se forme un trajet qui court en filigrane mais ne s'impose pas à première vue : le spectateur peut l'ignorer ou, au contraire, le mettre en jeu, s'il prend la peine de suivre son parcours » (André 5).

Conformément à ses analogies avec la tradition biblique, la «nuit », dans le film, semble receler une ambiguïté radicale. Présentée vers l'ouverture comme paysage des ténèbres ontologiques, sa représentation se complexifie par la suite à mesure que la lumière s'immisce dans l'espace urbain. On propose de sonder cette ambivalence en rattachant 
le motif à un thème central, celui de l'amour. On exposera tout d'abord le thème, avant d'en venir ensuite aux différentes acceptions de la «nuit». De cette manière, l'on espère mettre au jour la valeur esthétique et morale de Knight of Cups.

\section{Le thème de l'amour}

4 Le héros du film, Rick (Christian Bale), est un scénariste à succès de Hollywood en proie à une crise existentielle. Cumulant des aventures avec plusieurs femmes, celui-ci déambule à intervalles dans Los Angeles, à pied sur la plage ou à bord de son coupé de luxe, apparemment en recherche d'un objet qu'il peine à identifier. Au fil du récit, l'on comprend qu'il a renoncé, il y a longtemps, à l'amour que lui proposait Nancy (Cate Blanchett), qu'il continue de côtoyer et dont la présence à ses côtés contraste fortement avec les relations tumultueuses qu'il entretient par ailleurs avec les autres femmes du film.

5 Le thème de l'amour est abordé par le truchement de plusieurs sources qui s'insèrent toutes dans la tradition platonico-chrétienne. La première jonction est établie avec le Chant de la Perle, le plus célèbre poème de la littérature syriaque ${ }^{2}$. Ce texte est évoqué au début du film par le père du héros, qui parle alors dans la conscience du fils pour l'inviter à se remémorer une fable qu'il lui aurait contée dans son enfance ${ }^{3}$. "Remember the story I used to tell you when you were a boy ", lui dit-il, « about a young prince-a knightsent by his father, the king of the East, West, into Egypt, to find a pearl ». Tandis que le père paraphrase la suite du poème, racontant comment, une fois le prince arrivé en Égypte, les habitants lui servirent une coupe qui lui ôta la mémoire et le plongea dans un profond sommeil, Rick est entraîné à une fête costumée sur la terrasse d'un appartement, où deux jeunes femmes lui glissent entre les mains une coupelle qui le plonge dans l'ivresse.

6 "The king didn't forget his son ", poursuit le père quand, à l'aube, Rick regarde la ville à travers un garde-corps en verre. "He continued to send words, messengers, guides." Montrant le héros qui regarde vers le lointain urbain, un plan laisse apparaître à ses côtés, inaperçue de lui, une jeune femme encore vêtue du costume d'ange qu'elle avait revêtu pour la fête, et dont la présence à l'écran dialogue avec les «messagers » et les "guides » évoqués par le père hors-champ. Au plein de l'ivresse, la caméra s'était approchée des ailes qu'elle portait dans son dos, ce qui avait déclenché des cris de mouettes sur la bande sonore, comme en rappel des origines oubliées et de la possibilité de l'envol.

7 La seconde référence qui établit le lien avec ce thème est Le Cinéphile (The Moviegoer, 1961) de Walker Percy. Après avoir envisagé de porter le texte à l'écran dans les années 80, le cinéaste semble être revenu en partie sur ce projet avec Knight of Cups. On peut en effet considérer que le film constitue une adaptation très libre de l'ouvrage ${ }^{4}$. Rédigé à la première personne sous la forme du «courant de conscience» de la littérature moderniste, The Moviegoer retrace le quotidien de Binx Bolling, un agent de change annihilé par l'expérience de la guerre. Peinant à établir des relations durables avec les femmes, celui-ci tente de fuir les problèmes de sa vie en se rendant la nuit au cinéma. Un jour, le protagoniste est arraché à sa routine quotidienne, saisi du sentiment de ce qu'il nomme une "recherche », et entame un voyage symbolique qui le conduit de la Nouvelle Orléans (Louisiane) à Chicago (Illinois), en quête d'un sens pour sa vie. 
Influencé par la philosophie existentialiste, le livre de Percy s'ouvre par une citation ${ }^{5}$ du Traité du désespoir (1849) de Søren Kierkegaard ${ }^{6}$. Le philosophe situe l'existence humaine à la croisée des chemins, avec la nécessité d'opérer un choix et la possibilité d'une transfiguration ontologique. Dans son ouvrage Ou bien... ou bien (1843), Kierkegaard évoque deux manières distinctes d'être au monde qu'il nomme respectivement les "stades" (ou "sphères») esthétique et éthique. Alors que le premier évoque un mode d'être immédiat à l'art et à l'amour, où seules comptent les joies de la conquête et la jouissance de l'instant, le second stade ouvre la voie à une crise existentielle, au cours de laquelle le moi prend conscience de la nécessité d'une recherche intérieure et d'un lien à rétablir avec le transcendant. À l'issue de sa quête, s'il l'entreprend, le moi qui désespère renoue avec lui-même et ce qui se trouve hors de lui, il "plonge, à travers sa propre transparence, dans la puissance qui l'a posé » (Kierkegaard 1988, 63); ce faisant il rétablit le lien à soi et à l'en soi, il découvre le vrai amour, la parenté qui l'unit au monde. Par-delà l'éthique, le moi entre alors dans la sphère ultime, le religieux, que Kierkegaard rattache à une figure inspirée du Moyen Âge, le « chevalier de la foi »".

9 Si le titre de Knight of Cups $^{8}$ n'est pas sans rappeler cette dernière figure (Logan 2016), les sphères de l'esthétique et de la foi semblent trouver des échos notables dans le film. La première serait représentée par le séducteur Tonio (Antonio Banderas), qui souffle au héros l'adage "empiriste» (Berthomieu 2015, 10) : "There are no principles; just circumstances » et l'encourage à choisir une fille, lui dit-il, comme l'on choisirait un parfum de glace. Le royaume de ce personnage, introduit par des plans sous-marins où des corps «souillent» apparemment les eaux d'une piscine de leur pesanteur (Berthomieu 2015, 10), se situe a priori du côté de la pure décadence. Le film s'attache toutefois à parasiter ce symbolisme, le rôle de Tonio lui-même s'avérant en réalité très ambigu.

10 C'est par des biais esthétiques, justement, que le film met en place cette tension entre désir et spiritualité, en particulier lors d'une scène qui se déroule dans les intérieurs luxueux de la villa du séducteur. Tandis que les cordes de The Pilgrim's Progress ${ }^{9}$ convoquent discrètement en fond sonore le thème du voyage du soi, Rick se tient près d'une fenêtre, jouant des doigts avec de petites perles accrochées à un rideau qui rappellent la "perle» du chant syriaque. La texture soyeuse de l'étoffe trouve immédiatement écho, aux plans suivants, avec les robes de deux femmes qui lui font face: l'une vêtue de rose, l'autre de noir, cette dernière attirant l'attention du protagoniste sur son verni à ongles pour lui demander s'il aime la couleur rose.

Plutôt que de condamner les attitudes superficielles du monde qu'il porte à l'écran (comme le fera Tonio à l'issue de cette séquence : "The world is a swamp. You have to fly over it»), le film exploite une parenté inattendue entre le motif de la "perle» et les figures féminines autour de Rick. La voix de Tonio, entendue hors champ, se charge simultanément de l'initier à cette ambiguïté esthétique. "I love the company of women ", dit-il comme en conversation dans les environs. "It seemed they knew something, but closer-to the mystery. » Pendant ces mots, la caméra, très mobile, s'approche de la main vernie de la femme en noir, puis recule pour élargir la vue, plaçant systématiquement au centre du champ, non la main de celle-ci, mais la femme vêtue de rose, la couleur du verni à ongles. Deux plans successifs l'isolent finalement, tournant le dos au héros pour révéler à ses regards ébahis une paire d'ailes tatouées au bas de sa nuque, entre lesquelles figure le mot évocateur « faith $»^{10}$. 

lecture enregistrée du Phèdre par Charles Laughton, d'après une traduction libre et poétique de l'écrivain américain Christopher Isherwood ${ }^{12}$. Accompagné de l'orgue du Miserere $^{13}$ d'Arvo Pärt, l'acteur interprète hors champ le passage central du livre, où Socrate évoque le voyage de l'âme et sa chute dans l'incarnation, traduit comme suit :

Once the soul was perfect and had wings,

It could soar into Heaven where only creatures with wings can be, But the soul lost its wings and fell to Earth,

And there it took an earthly body.

15 À l'écran, Rick conduit son coupé de luxe au côté de Della (Imogen Poots), la première héroïne du film, qui va se charger d'endosser le rôle d'un guide esthétique pour le héros. Quand la voix hors champ évoque la chute de l'âme dans l'incarnation, le montage donne lieu à un écran noir (coïncidant avec le mot «Earth»), puis un fondu inversé révèle, suspendus au plafond d'une chambre de l'Aquarium du Pacifique, à Long Beach, en Californie, les corps d'une baleine bleue et de son petit. Ce plan fait doublement « entrer la lumière » dans le monde terrestre et diégétique : grâce à l'effet de fondu inversé d'une part, qui transmute le fond noir en photographie (l'écriture avec la lumière selon le sens étymologique), et grâce aux rayons du soleil d'autre part, qui pénètrent à l'intérieur de l'Aquarium par de larges vitres à l'arrière-plan. Suspendus dans la lumière, les corps des cétacés portent à l'écran l'ambivalence de l'incarnation, entre pesanteur et légèreté, tandis que l'Aquarium qui s'illumine aux sons de l'orgue semble un lieu à la fois sacré et naturel, une cathédrale aquatique, à l'échelle de la création ${ }^{14}$.

Par la suite de l'extrait, le héros contemple les poissons dans les bassins de l'Aquarium. Charles Laughton poursuit au sujet de l'âme :

Now, while it lives in this body no outward sign of wings can be seen;

Yet the roots of its wings are still there,

And the nature of wings is to try to raise the earthbound body and soar with it into Heaven. 
Face à Rick, une raie filmée en contre-plongée s'élève dans la lumière telle l'incarnation de la légèreté à laquelle il aspire. À ce point le cinéaste opère une coupe dans l'enregistrement pour le reprendre au moment où Socrate raconte comment, grâce à l'amour terrestre, l'âme peut renouer avec la beauté qu'elle contemplait parmi les cieux :

When we see a beautiful woman, or a man,

The soul remembers the beauty it used to know in Heaven.

Pendant la lecture du premier vers, comme mue par un élan concerté avec l'enregistrement, la caméra vient chercher l'héroïne qui s'éloigne parmi les visiteurs : dans son dos, le gilet qu'elle porte dessine deux fentes qui lui font deux ailes. Lors de la lecture du second vers, Rick vient se placer entre deux enfants (Malick s'est arrangé pour que ceux-ci soient présents en grand nombre dans les couloirs de l'Aquarium) en train de s'émerveiller face aux poissons, et dont l'apparition coïncide avec le mot « Heaven ». Dans le contexte symbolique du film, ceux-ci semblent deux "messagers", deux « petits anges » dépêchés de leur royaume pour convoquer à la mémoire du héros la pureté oubliée des origines ${ }^{15}$.

On envisagera que cette séquence puisse constituer le «moment favori» de Knight of Cups, d'après la définition musicale appliquée par Emmanuelle André au cinéma. Survenant peu après le début du film, un tel moment représente "une trouée, une percée » qui, à la fois, résumerait l'aspiration de l'œuvre et serait susceptible de la contaminer (André 37). Le moment favori constituerait ainsi « un bref passage qui fait sens parce que justement il contiendrait en partie les problèmes posés par le film. Il les explorerait d'une part, les déplierait d'autre part, jusqu'à faire se confronter le film à ses questionnements essentiels» (André 38). On verra en effet que le film mitige progressivement sa représentation de la «nuit» en y intégrant l'esthétique de l'Aquarium (l'orgue du Miserere, la lumière et la voix de Charles Laughton).

L'idée qui nous guidera est que le film exploite une représentation romantique de la « nuit $\aleph^{16}$, en portant à l'écran, par le truchement du monde urbain, l'intériorité du protagoniste, ses "paysages intimes " ou encore ses "choses mentales", entrevues dans «l'œil de l'esprit $»^{17}$. Malick semble en outre associer le motif au thème de la déambulation : la «nuit " y serait une trajectoire, à la façon dont les "rêveries» de Rousseau représentent un cheminement spirituel depuis la condition de l'exil jusqu'au sentiment de la transcendance.

\section{Nuit urbaine et désespoir}

21 La «nuit » intervient pour la première fois quelques minutes après le début du film, alors que le héros déambule seul dans les «backlots» de la Warner, à Hollywood ${ }^{18}$. Quand les portes d'un hangar se referment sur un ciel diurne, privant littéralement l'écran de la lumière du jour, la diégèse bascule dans la «nuit " et une composition musicale est entendue : La Mort d'Ås ${ }^{19}$ du compositeur norvégien Edvard Grieg, dont la présence convoque implicitement le contexte de sa création. Intégrée à la Suite $n^{\circ} 1, O p$. 46 en 1888, la composition fait à l'origine partie de la musique de scène écrite par le musicien pour le drame philosophique de Henrik Ibsen, Peer Gynt ${ }^{20}$. Comme son titre l'indique, La Mort d'Åse accompagne dans la pièce le moment tragique de la mort d'Åse, la mère du héros (à la fin de l'acte III). Dans cette scène très poétique, Peer Gynt se trouve au chevet de sa mère et lui raconte son envolée sur un traîneau vers le château 
merveilleux de Soria-Moria ${ }^{21}$. D'inspiration romantique, jouée en si mineur doloroso par un orchestre symphonique, $L a$ Mort d'Åse instaure une atmosphère mélancolique qui permet à Malick d'associer la nuit urbaine au désespoir.

Lorsqu'une vue prise depuis un toit d'immeuble s'avance vers la façade du Los Angeles Theater, la voix du père est entendue, filant l'allégorie du Chant de la Perle : «'Can't figure your life out ", dit-il au fils. À l'écran, la façade de l'édifice réfléchit sur le contexte théâtral de La Mort d'Åse, et introduit la métaphore de la déambulation du soi sur la scène du monde. Prolongeant immédiatement le thème, un plan filme le héros qui marche au sommet d'un immeuble sur un fond de ciel nocturne et de gratte-ciel. Les mots du père : " 'Can't put the pieces together » résonnent des édifices éparpillés dans la profondeur de champ, qui semblent autant de fragments ontologiques, une «terre vaine $^{22}$ » intérieure. Sur ce fond nocturne, le protagoniste escalade un à un, au rythme lent et dramatique de La Mort d'Åse, les petits promontoires de béton qui jalonnent son passage, s'élevant et retombant tour à tour dans la "nuit», comme une âme nostalgique des hauteurs mais que le corps priverait de l'ascension.

Cet aspect se traduit en termes formels. À l'écran, le corps du héros se résume à un aplat noir, tandis qu'à l'arrière-plan la métropole lui fournit un fond lumineux, un "backlighting" qui le fait apparaître en silhouette et, tel un écran, s'ouvre en tant qu'espace de projection. Dans la première partie d'Ou bien... ou bien, Kierkegaard évoque des figures littéraires de la tristesse profonde, qu'il nomme "silhouettes" parce qu'elles se trouvent «du côté sombre de la vie et aussi parce que, en tant que silhouettes, elles ne sont pas immédiatement visibles » (2015 160). Tout en les excluant de la représentation plastique pour en faire l'objet de la poésie et de la psychologie, le philosophe recourt à une métaphore qui, en 1843, au moment où naît la photographie, préfigure le dispositif du cinéma. "Pour la voir ", écrit-il, «il me faut d'abord la tenir devant le mur et considérer, non pas l'image immédiate, mais sa projection» (2015 160). Ses descriptions auraient pu lui être inspirées par une toile d'Edward Hopper : « On suit la rue; les maisons se ressemblent, et seul l'observateur exercé soupçonne que celle-ci prend à minuit un aspect tout différent alors qu'y déambule un infortuné qui n'a pas trouvé le repos; il gravit l'escalier, son pas résonne dans le silence de la nuit. » (2015 162)

24 Le lien entre la silhouette humaine et le cinéma, c'est Stanley Cavell, l'ancien professeur de Malick, qui l'établit dans La Projection du monde (The World Viewed, 1971, 1979) ${ }^{23}$ - livre lu et commenté à sa demande par Malick -, au sujet des Moissons du ciel (Days of Heaven, 1978): "Des objets projetés sur un écran sont par essence réflexifs », écrit-il, «ils se produisent comme auto-référentiels, réfléchissant sur leur origine physique. Leur présence renvoie à leur absence, à leur situation en un autre lieu.» (Cavell 1999, 12) Or l'esthétique de Malick exploite cette ambiguïté ontologique : de même que les silhouettes de Kierkegaard et la projection selon Cavell présentent une réalité paradoxale, manifeste sur un fond d'absence, la ville nocturne autour de Rick devient une «indication télégraphique " de ses «arcanes profonds " (Kierkegaard 2015, 163). En retour de ce transfert ontologique, le « backlighting » l'évide de toute expressivité pour le produire en négatif, c'est-à-dire, ni plus ni moins, en spectre.

L'action est ensuite transposée sur l'autoroute de la ville, où des contre-plongées filment, depuis la voiture du protagoniste, les gratte-ciel qui s'élèvent sous un ciel nocturne. Pendant ces vues, le père dit hors champ : «A pilgrim on this earth, a stranger », 
usant d'un lexique biblique qui tend à associer la modernité à une terre d'errance ${ }^{24}$. Concordant avec ce langage et le thème dramatique de La Mort d'Åse, ces vues de la ville semblent produire à l'écran une cité sous-terraine, formidable dans ses dimensions mais privée de la clarté originelle. Les réfractions qui s'établissent à la surface des édifices, où se reflètent comme en autant de miroirs troubles la ville et le ciel noir, fournissent des équivalents visuels au passage de Saint Paul, dans l'épitre aux Corinthiens, décrivant le monde comme un lieu où le transcendant n'est perçu « qu'au moyen d'un miroir, d'une manière obscure ${ }^{25} »(1 \mathrm{Co}, 13: 12)$.

Dans le même ordre d'idée, la jungle d'édifices présente à l'écran un simulacre des palmiers de Los Angeles, qui furent isolés explicitement dans la séquence précédente, lorsque la caméra avait panoté vers les arbres et que Rick s'était adressé au spectateur en ces termes: "You see the palm trees? They tell you anything's possible». Ces derniers réapparaitront dans le cours du récit, filmés par des contre-plongées sur un fond de ciel azuréen (en particulier quand Charles Laughton interprétera une phrase du Phèdre sur les ailes de l'âme). Si ces palmiers symbolisent la possibilité de l'envol, les gratte-ciel tels qu'ils apparaissent dans cette séquence, excédant le champ de toute part et voilant jusqu'au ciel de nuit, confèrent au monde urbain un caractère écrasant et paraissent conjurer toute transcendance.

Dans la tradition américaine, ces contre-plongées rappellent par exemple City $\mathrm{Night}^{26} \mathrm{de}$ Georgia O'Keeffe, où l'on retrouve une composition plastique semblable : les gratte-ciel new-yorkais, présentés en majesté depuis les rues, y sont si grands qu'ils excèdent les limites du cadre, occupant la plus large partie du ciel et dominant jusqu'à la lune même qui en est réduite à figurer au bas de la toile. On verra que le rapprochement avec cette toile s'avérera particulièrement utile lorsqu'il s'agira de mitiger le rapport d'opposition que le film paraît établir ici entre la nature et la métropole.

Par la suite de l'extrait, la "nuit» semble momentanément conjurée quand le héros entre dans un tunnel où il se trouve baigné dans la lumière électrique. Cette entrée coïncide avec le moment où La Mort d'Åse reprend son thème à la quinte supérieure (il s'agit d'une envolée). Dans le drame d'Ibsen, cette reprise lyrique accompagne le passage où Peer Gynt raconte à sa mère sa réception au château de Soria-Moria par saint Pierre et l'Éternel. L'évocation céleste se retrouve en un sens à l'écran avec la lumière d'or qui inonde le héros, renvoyant par métonymie aux rayons du soleil. L'envolée, pourtant, «tombe à plat »: car de même que l'apogée de la composition porte à son point culminant la profonde tristesse du drame d'Ibsen (c'est le moment où la mort d'Åse est sur le point de mettre un terme à la rêverie de Peer Gynt), de même, l'éclairage du tunnel signale sa dimension d'artifice.

Horizontal et circonscrit, sans lien réel avec le transcendant - quand le soleil, chez Malick, en est le symbole -, la lumière du tunnel ne retient que l'image de son modèle, non sa substance où résiderait son sens profond. Pour le dire dans les termes de la scolastique, la lumière phénoménale (appelée lumen) ne renvoie ici à aucune lumière essentielle (lux), laquelle seule permettrait d'établir le lien entre l'espace diaphane que traverse le héros et le transcendantal ${ }^{27}$. Tandis que cet éclairage électrique baigne Rick dans une lumière d'or, il fait " écran » aux cieux. Fermant l'accès à la réalité extérieure, le tunnel s'ouvre à son tour en tant qu'espace de projection, couloir mental où s'expriment les fantasmes et désirs contrariés de l'envol. Au lieu de s'élever comme le suggère l'ascension lyrique de La Mort d'Åse, le héros plane ainsi dans un ciel artificiel, côtoyant les cieux seulement par abstraction. 
30 L'action est finalement déplacée dans l'appartement du protagoniste, où deux plans filment, depuis des balcons, la ville nocturne et ses espaces fragmentés (extérieurs quadrillés par des réseaux de routes et de fils téléphoniques, intérieurs cubiques où les corps humains se meuvent en silhouettes). Pendant ces plans, le père dit dans la conscience du fils: "fragments, pieces-of a man", confirmant la relation que la modernité entretient, dans sa représentation formelle, avec le désespoir ${ }^{28}$. Le langage même du père, discontinu et paratactique, tend à induire une relation de cause à effet entre les fragments tristes et éparpillés de la ville nocturne et la "forme de vie ${ }^{29}$ " humaine. Comme s'il fallait comprendre que ce monde, exilant les hommes de la nature, du transcendant, réduisait leurs vies en pièces.

31 Dans le même temps, le film semble inviter à porter un autre regard sur la modernité. Au sein de cette séquence, les plans des gratte-ciel depuis l'autoroute ont filmé la plus haute tour de Los Angeles, couronnée d'un cercle lumineux. La tonalité en si mineur et la cadence doloroso de La Mort d'Åse, combinées aux interventions du père, font d'abord apparaître cet édifice comme le symbole d'un royaume décadent, comparable à la cité de Metropolis (1927) de Fritz Lang, nommée par un carton-titre «nouvelle Babel $»^{30}$. Son halo de lumière blanche, surplombant la ville nocturne à la place de la lune, et l'attraction que cette tour paraît exercer sur la caméra qui la place a plusieurs reprises au centre du champ - comme si elle pressentait dans cette couronne lumineuse un indice de la clarté originelle -, tendent toutefois à l'investir d'une valeur sublime ${ }^{31}$. La comparaison se maintiendrait alors d'autant avec la toile d'O'Keeffe, où l'on retrouve une ambiguïté analogue avec la tour blanche et lumineuse à l'arrière-plan, derrière les deux gratte-ciel sombres, dont la présence réfléchit sur celle de la lune au bas de la toile. On propose de montrer comment les représentations ultérieures de la nuit urbaine, dans le film, exploitent cette tension et invitent à mitiger le rapport de contradiction entre la modernité et le spirituel.

\section{Parmi les ténèbres, la lumière}

32 Tournées pour l'essentiel sur les routes de la Californie ou dans des clubs, les «nuits » ultérieures du film exploitent des tensions entre l'obscurité inhérente à ces espaces et la présence soit de la lumière elle-même (parfois lunaire, le plus souvent électrique) soit de ses symboles (objets volants, paires d'ailes, figures en lévitation, plumes tombant du plafond, etc.). On rendra compte de ces tensions en étudiant quatre occurrences particulières, qui font groupe de par un dispositif commun de mise en scène où une voix et un thème musical «hors champ » dialoguent chaque fois avec l'esthétique visuelle du film. On verra que ces modulations donneront lieu à un glissement radical dans la représentation du motif.

33 La première de ces «nuits » intervient alors que le héros se trouve à nouveau sur le toit d'immeuble en face du Los Angeles Theater, au côté de son père et de son frère. Diurne tout d'abord, la scène se déroule sous un ciel opaque qui s'accorde avec la désolation urbaine autour des personnages (bâtiments grisâtres, murs fissurés, arrière-cours obstruées). Entendu hors champ, le père établit une fois encore la jonction entre cet espace symbolique et le thème du désespoir. "I suppose that's what damnation is », dit-il tandis que la caméra plonge, depuis le point de vue de Rick, sur l'espace vide et géométrique d'une arrière-cour. "The pieces of your life never to come together." 
Convoquée par ce rappel du désespoir, la «nuit » entre en scène à mesure que le protagoniste conduit sa voiture sur les routes de la métropole.

Parallèlement, la bande sonore se peuple des nappes ambiantes du morceau Hyperborea (1997) de Biosphere (nom de scène du musicien norvégien Geir Jenssen), s'accompagnant bientôt d'une voix masculine, intégrée par le musicien à l'enregistrement: il s'agit de celle du Major Briggs (Don S. Davis) dans le premier épisode de la seconde saison de Twin Peaks ${ }^{32}$, rapportant à son fils une "vision " qu'il aurait eue dans son sommeil. Cette seconde voix paternelle « hors champ » est rendue partiellement inintelligible du fait des distorsions sonores opérées par Biosphere, et résonne ainsi comme une voix indistincte, entendue depuis les profondeurs d'un songe. "This was a vision ", distingue-t-on à peine en fond sonore, "fresh and clear as a mountain stream ». Puis plus audible : «The mind revealing itself to itself ».

Pendant ces mots, des plans filment les slogans et icônes de la société de consommation autour de Rick, restituant à l'écran un monde frénétique et superficiel, froid « comme du marbre » selon les mots du chef décorateur Jack Fisk (Sragow 2016), qui contraste fortement avec la vision claire et naturelle de l'enregistrement. Une relation ambiguë s'établit toutefois entre la ville nocturne et les descriptions de la voix hors-champ lorsque cette dernière en vient à évoquer un "palace aux proportions fantastiques, d'où semblait émaner une lumière de l'intérieur de son marbre étincelant et radieux ». La caméra s'élève en effet invariablement, depuis la voiture de Rick, vers les panneaux publicitaires qui dominent les bords de la route, pour opérer un zoom avant - un mouvement d'objectif presque absent de l'œuvre de Malick, proscrit par un "dogme » établi avec les techniciens ${ }^{33}$ - et isoler les visages lumineux des actrices et des modèles sur les immenses panneaux.

Comme mue par un élan extérieur à la conscience du protagoniste, la caméra semble rediriger son attention vers une signification que recèleraient, quoique sur un mode obscur, les stéréotypes de la modernité. Résonnant de la lumière de l'enregistrement qui émane d'un "marbre étincelant et radieux ", ces visages féminins représentent à l'écran des aplats clairs et lumineux qui tranchent sur le ciel nocturne. Avec ces panneaux publicitaires, la forme humaine cesse momentanément de hanter la ville en spectre. Filmés sur un fond de ciel de nuit, leur fonction semble être d'orienter le regard vers la zone obscure et de l'éclairer.

Replacés dans le contexte diégétique, ces visages rappellent les femmes que côtoie le protagoniste et renvoient au thème de l'amour. Un lien s'établit, en particulier, entre ces figures féminines, illuminant le ciel, et les mots du Phèdre cités plus tôt par Charles Laughton, selon lesquels « lorsque nous voyons une belle femme, l'âme se remémore la beauté qu'elle contemplait jadis parmi les cieux ». Sans rien expliciter, ce jeu de clair/ obscur ouvre la voie à une modulation, à un glissement dans la représentation de la " nuit». Bien qu'obscur, le monde moderne pourrait receler dans sa physionomie un élément contrastant, à même de convertir la «nuit» en une vision «neuve et claire comme un cours de montagne " (pour reprendre encore une fois l'enregistrement), où l'esprit se révélerait à lui-même.

38 Cette tension est explorée un quart d'heure plus tard dans le film, à travers une suite d'épisodes nocturnes. L'hindouiste Helen (Freida Pinto), femme spirituelle au parfum d'Orient qui rappelle les origines du Prince dans le Chant de la Perle ${ }^{34}$, vient d'inviter le héros à nouer avec elle une relation amicale. Accompagnée de La Chanson de Solveig (autre fragment de la musique de scène composée par Grieg pour Peer Gynt ${ }^{35}$, intégré en 
1891 à la Suite $n^{\circ} 2,0 p$. 55), celle-ci se dérobe joyeusement à ses étreintes et lui murmure : «Dreams are nice, but you can't live in them ».

Le héros peine toutefois à comprendre son message et la jeune femme finit par lui refermer sa porte après qu'il l'ait raccompagnée chez elle. Rick entame alors une nouvelle virée nocturne qui le conduit à un club de strip-tease où il fait la rencontre de Karen (Teresa Palmer), la «Grande Prêtresse ${ }^{36}$ » des lieux. Son univers à elle, à l'opposé du monde spirituel de Helen, est un royaume du corps d'illusion, où l'humaine se réduit à un écran des fantasmes et à un objet de consommation - à l'image de ces parcelles de corps synthétiques et presque morbides qu'un plan filme à l'entrée du club derrière des vitrines. L'ambiance sonore du lieu retranscrit cette idée, avec ses nappes électroniques monotones, horizontales, où des gémissements répétés viennent clamer l'hégémonie du désir et le triomphe de l'instant.

Dans ce temple de la chair où l'âme n'est qu'un mirage, hommes et femmes se racontent de fausses histoires et jouent à endosser des rôles comme sur la scène d'un Theatrum mundi ${ }^{37}$. Cette parade du soi s'accompagne d'une négation du transcendant par Karen, qui chuchote, bien explicite, au héros : "There's no shuch thing as forever. The highs don't add up » Le club de strip-tease rappelle alors l'épisode biblique du Veau d'or tel qu'ont pu le porter à l'écran Cecil B. DeMille dans Les Dix Commandements (The Ten Commandments, 1956) et King Vidor avec les Égyptiens de Salomon et la reine de Saba (Solomon and Sheba, 1959), où l'idolâtrie et la passion de la chair sont figurées comme un carnaval nocturne inquiétant.

41 Le thème de la «nuit » en tant qu'illusion culmine lorsque Rick et Karen se rendent à Las Vegas. Si, au début du film, la jeune Della conduit Rick à l'Aquarium du Pacifique pour qu'il contemple dans les eaux la légèreté et la lumière, Karen le plonge au contraire dans un monde artificiel, que le film ne nomme pas exactement Babylone mais - par le truchement de l'opéra de Williams où l'acteur John Gielgud lit un passage du Voyage du Pèlerin - «donjon fétide $»^{38}$. De ce lieu, absolument tout ce qui apparaît à l'écran tient de l'imitation, depuis les pyramides d'Égypte, les temples grecs, la statue de la Liberté, la tour Eiffel et la Victoire de Samothrace ${ }^{39}$ jusqu'aux cieux mêmes qu'un plan filme peints sous la coupole d'un centre commercial telle une pâle évocation du firmament de la Chapelle Sixtine.

42 La représentation nocturne s'y avère pourtant profondément ambiguë, en se mitigeant d'éléments associés précédemment au monde de l'Aquarium. Tandis que le couple roule dans la nuit, l'orgue du Miserere de Pärt intervient pour la seconde fois, convoquant le thème de la cathédrale cosmique (celle de l'Aquarium, où le monde phénoménal était irradié par la lumière). Cette première analogie se renforce de l'usage d'un second enregistrement de Charles Laughton, où l'acteur interprète des extraits du Psaume $104^{40}$. Entendue hors-champ sous la forme de bribes, cette voix s'évanouit après quelques secondes à peine, semblable elle aussi à un appel indistinct, à une voix de conscience perçue dans les profondeurs de la «nuit $»^{41}$. Alors que le texte biblique entre apparemment en contradiction avec le monde présenté à l'écran (le psalmiste y évoque la Création de la Terre par l'Éternel), la mise en scène s'attache à opacifier cette distinction. «...Who laid the foundations of the Earth» entend-on lorsque la voiture de Rick traverse un tunnel obscur, «that it should not be removed for ever ». Dans la partie supérieure du champ (celle que la tradition platonico-chrétienne associe au spirituel), des ampoules électriques jalonnent en ligne droite la voûte du tunnel jusqu'à sa sortie, tandis qu'au 
bas de l'image (la partie associée au matériel par la même tradition), la croix de la Mercedes de Rick s'avance sur la route sombre tel un Christ en pèlerinage dans le désert urbain. Quand une coupe transpose la scène sur l'autoroute nocturne, la ligne tracée par l'éclairage au plafond du tunnel se prolonge avec un faisceau lumineux qui jaillit dans un ciel de nuit. À bord de la voiture, la caméra s'élève d'abord vers la pointe du rayon pour s'abaisser et révéler sa source: le sommet d'une fausse pyramide d'Égypte, filmée sous la pleine lune.

Dialoguant implicitement avec cette composition plastique, la voix hors champ raconte qu'à l'origine, le Créateur écoula « les eaux stagnantes au-dessus des montagnes » afin qu'elles se répandissent dans les vallées, et fit pousser les herbes pour le bétail et les plantes pour le besoin des hommes. À l'écran, le ciel de nuit qui surplombe la pyramide semble cette mer stagnante appelée à s'écouler pour révéler le monde dans son harmonie originelle. Simultanément, l'éclairage naturel et zénithal de la lune trouve un pendant inversé, une sorte de réflexion, dans le faisceau de la pyramide qui, tel le SaintJean-Baptiste ${ }^{42}$ de Léonard de Vinci, indique le hors-champ. Le trope est décliné avec les deux contre-plongées suivantes, lesquelles présentent successivement sur le fond du ciel de nuit la statue de la Liberté, torche brandie dans l'obscurité, et la flèche éclairée de la tour Eiffel. Tandis que les prises de vue et la ligne de fuite des deux structures indiquent les cieux, un raccord avec le faisceau de la pyramide confirme l'analogie avec le hors-champ, avec l'espace diurne, la lumière.

Enfin, le thème est explicité avec une statue d'ange, filmée de dos, les ailes à l'avantplan, et dont les « cornes » (les rayons lumineux) ainsi que la trompette d'or évoquent l'archange Gabriel, annonçant la seconde venue du Christ sur la Terre. Cette apparition angélique coïncide en outre avec l'apparition, dans le Miserere, d'une partition de clarinette, laquelle exploite une nouvelle résonnance entre la bande sonore et le monde à l'écran. À l'arrière-plan, jouxtant virtuellement les ailes de la statue, la pleine lune brille dans la nuit. Celle-ci n'entre pas en contradiction avec la ville nocturne, mais se mêle au contraire aux formes urbaines, dont les éclairages artificiels lui fournissent même d'étonnants pendants (en particulier la lanterne d'un réverbère, le sommet un peu ovale de la tour Eiffel et une fausse lune au sommet d'un immeuble, disposés respectivement au centre et des deux côtés du champ) ${ }^{43}$.

46 À l'heure nocturne, la ville illuminée semble rétablir le lien avec le merveilleux que ses espaces diurnes voilaient au regard. Plutôt que de renvoyer au jour, la «nuit» recèlerait alors une fonction essentielle : réactiver, réenchanter une relation ternie au monde. Si certaines peintres de nuit ont tenté de réintroduire le merveilleux dans l'espace urbain, Malick pourrait se rapprocher plus encore de ces autres artistes du nocturne tels que Steichen avec Brooklyn Bridge $e^{44}$, où les édifices et l'éclairage urbain prennent une valeur symbolique (Valance 306-9). Au lieu d'adoucir ou de magnifier la ville en lui conférant une valeur intrinsèque, la «nuit », chez Malick, rétablirait le lien perdu avec le transcendant.

Cette acception du motif est explicitée vers la fin du film, lorsque le héros déambule dans le désert, accompagné de La Mort d'Åse. Une nouvelle voix masculine se charge alors de prendre le relais du père et de ses avatars : parlant avec un accent allemand, cette voix va en effet s'avérer être celle d'un " père » spirituel, un prêtre. En tant que telle, son intervention clarifie ce que le film avait jusqu'ici mis en scène à travers des jeux visuels de clair/obscur, des citations musicales et des interventions verbales largement énigmatiques. 

de la «nuit », comme agent de la rédemption, en des termes proprement filmiques. Une jeune femme blonde particulièrement énigmatique (Isabel Lucas) y accompagne le héros dans le désert, confirmant l'affirmation du théologien selon laquelle, dans son errance, Rick n'est pas seul. De cette figure mystérieuse, interprétée par une actrice très svelte et gracieuse, Malick ne filme jamais distinctement le visage, ce qui tend à l'associer à un ange ${ }^{46}$. Quand, peu avant la tombée de la nuit, celle-ci conduit le héros près d'un champ d'éoliennes, le père intervient hors champ : « Find the light you knew in the East », dit-il au fils en référence au Chant de la Perle, " as a child ». Sa voix s'inscrit sur le fond presque assourdissant du vrombissement des éoliennes, lequel fournit une version moderne, mécanique du « souffle». Tandis que la nuit s'annonce, ces structures représentent à l'écran une colonie d'étoiles et réfléchissent, à l'heure nocturne, sur l'éclat des cieux. «The moon », explicite le père, « the stars... ».

"'Seems you're alone», entend-on pendant que Rick marche seul dans le désert. Aux mots : "you're not ", la caméra se détourne du héros pour filmer le soleil derrière un nuage, motif visuel dont l'apparition occasionne sur la bande-son un souffle venteux ${ }^{45}$. À ce point, l'action est déplacée dans un appartement où, bien qu'il fasse encore jour, le héros et son père sont plongés dans l'obscurité par des rideaux tirés aux fenêtres. " Even now ", poursuit le prêtre, "He's taking your hand and guiding you, by a way you cannot see ». Tandis que les personnages apparaissent prostrés en figures de lamentation, la tête dans les mains et le regard rivé au sol, la caméra s'approche des rideaux clos ou entrouverts, indiquant, comme avec le nuage, la source lumineuse qui se trouve derrière.

désormais le prêtre (Armin Mueller-Stahl) en train de s'adresser au protagoniste. À l'arrière-plan, les rayons du soleil pénètrent à l'intérieur de l'édifice par des vitraux et placent le théologien en partie dans l'ombre, en partie dans la lumière. «To suffer binds you to something higher than yourself », poursuit-il face à la caméra, " higher than your own will. 'Takes you from the Earth, to find-what lies beyond it. » Quand avec ces derniers mots, le prêtre étend sa main dans la lumière, La Mort d'Åse reprend son thème à la quinte supérieure, évoquant le sublime, et le montage enchâsse des vues urbaines où le soleil point dans la profondeur de champ derrière la ville.

déformée sur les bords, l'idée d'un miroir trouble du transcendant, ces plans filment le quartier résidentiel d'une petite ville périurbaine, une métropole depuis l'autoroute et un cimetière. Près des tombes, la caméra plonge vers l'ombre d'un tronc sur le sol pour panoter vers l'arbre derrière lequel se devine, une fois encore, le soleil. La voix du prêtre continue d'être entendue hors champ, guidant le héros à l'instar du soleil qui, dans la profondeur, semble orienter la caméra. "We are not only to endure patiently the troubles He sends; we are to regard them as gifts-as gifts more precious than the happiness we wish for ourselves. "

Dans une pièce obscure, la jeune femme-guide tient une bougie entre ses mains. Les mots presque inaudibles qu'elle susurre au héros sur le thème de «la lumière dans l'obscurité » redirigent l'attention vers la bande-son, où le vrombissement mécanique des éoliennes vient de se changer en un chant de cigales ${ }^{47}$ et en un souffle venteux. " They serve you ", poursuit le père au sujet des astres pendant que le héros et la jeune femme marchent dans le désert, «they guide you on your way ». De façon significative, la 
scène est tournée à l'« heure bleue ». Exploitée abondamment par Malick dans La Balade sauvage (Badlands, 1973) et Les Moissons du ciel, celle-ci prend en l'occurrence une valeur symbolique quant au thème étudié. L'heure bleue désigne en effet le moment fugace entre le jour et la nuit où le soleil, bien qu'invisible derrière la ligne de l'horizon, inonde le paysage de sa lumière. Cet alliage de la nuit et du jour confère à l'atmosphère un chromatisme très particulier, à la fois bleuté et rougeoyant, propice, comme le note Jacques Aumont au sujet des Moissons du ciel, à des effets de «fantastique » (9).

Cette présence/absence réfléchit sur l'image filmique elle-même et sur son élément constitutif, la lumière, dont l'ambiguïté est précisément de n'être toujours perçue qu'indirectement, grâce à ses effets (Aumont 2010; Bouvrande 2016). Les lueurs de l'heure inscrivent autour des personnages une lumière phénoménale (lumen), traversant un espace diaphane mais doté de qualités esthétiques, le ciel, lequel renvoie à son tour à la source que l'on ne voit pas, la lumière " en soi ", le soleil (lux). De même que, dans la définition du cinéma par Cavell, la présence des choses à l'écran « renvoie à leur absence, à leur situation en un autre lieu » (Cavell 1999, 14), de même, les lueurs de l'heure bleue rendent compte d'une présence «hors champ", persistante en filigrane du voile nocturne. Enfin, tandis que le héros se trouve dans une grotte où brûle un feu, la jeune femme lui murmure depuis l'entrée : «darkness is not dark to you ${ }^{48}$ ». Bien que le protagoniste se trouve sous la terre, dans la partie la plus obscure du champ, le feu qui brûle à ses côtés atteste la persistance de la lumière, effectivement présente à ses côtés en dépit de son absence ${ }^{49}$. Introduite comme paysage du désespoir, la «nuit » en est devenue vecteur de la clarté.

\section{Conclusion}

Alors que la trajectoire de l'œuvre de Malick encourage dans un premier temps à y percevoir un antagonisme radical entre les grands espaces et la ville moderne, la représentation de la nuit urbaine dans Knight of Cups invite à reconsidérer cette opposition. On a tenté de montrer que le cinéaste puisait dans les traditions platonicochrétienne et romantique pour fournir une représentation mitigée de la modernité, où la « nuit » oscille entre expression du désespoir et manifestation du transcendant. Si la sensibilité de Malick à l'élément naturel prédomine, et si la ville, dans ses films, entre effectivement en tension très forte avec cette sensibilité, le cinéaste s'efforce toutefois de dépasser cette dichotomie pour proposer une réflexion originale sur la modernité et sur l'amour.

À l'issue de son ultime séquence nocturne, le film met en place sa résolution à travers une scène où la voix du père et l'enchaînement des plans apportent désormais un surplus de signification, une explicitation de la représentation parfois opaque que le film a produite de la nuit urbaine. Le père de Rick y paraphrase pour finir le Phèdre (255 $c-d$, Platon 131): "The light in the eyes of others ", tandis qu'à l'écran, Nancy, l'épouse divorcée de Rick, marche seule sur une plage et jette derrière elle quelques regards inquiets, comme à un époux lointain. Le père conclut par un chuchotement: "The pearl ». D'autres plans filment les gestes de tendresse que cette femme a sur le corps d'un homme meurtri de difformités dans un hôpital, et que la caméra, attentive ici aux formes de l'amour comme elle le fut à la lumière dans la "nuit ", filme de très près, indiquant leur importance. Ces gestes ne sont ni plus ni moins que les équivalents 
humains de la lumière qui s'est introduite dans la «nuit ». Ils suggèrent un raccord possible entre les hommes, et entre l'humanité et le cosmos.

La "perle» ontologique de Knight of Cups, c'est l'amour. Cette lueur scintille dans la forme humaine (inquiétude du regard, gestes tendres ou hésitants, bribes de langage) en réfraction de ces symboles cosmiques qui ont traversé le film comme autant de balises, de points de repères dans un continuum de ruine et de fragment. De cette analogie ressort un éloignement radical d'avec la philosophie de Platon: le corps humain et l'expressivité n'entrent pas pour Malick en contradiction avec l'esprit, mais l'accomplissent, le transmutent en formes et en sensations. La "nuit» serait alors porteuse d'une image, d'une idée, qui scintille obstinément dans l'obscur: le monde, malgré ses fissures et ses impuretés, reste un espace habitable, un lieu où il serait possible, pour ceux qui s'y engagent, d'aimer, d'être aimés.

\section{BIBLIOGRAPHIE}

André, Emmanuelle. Esthétique du motif : cinéma - musique - peinture. Paris : Presses Universitaires de Vincennes, 2007.

Aumont, Jacques. L'attrait de la lumière. Crisnée : Yellow Now, 2010.

Barnett, Christopher B. and Clark J. Elliston (ed.). Theology and the Films of Terrence Malick. New York: Routledge, 2016.

B, Benjamin. « Cosmic Questions ». The American Society of Cinematographers (août 2011). 1 er février 2020. http://www.theasc.com/ac_magazine/August2011/TheTreeofLife/page1.php.

Berthomieu, Pierre. «La question sans réponse. La spiritualité spectaculaire de Terrence Malick ». In Hollywood moderne : le Temps des voyants. Pertuis : Rouge profond, 2011. 653-668.

---. « 'Life Surpassing Itself' Terrence Malick, The Tree of Life et À la merveille: la fin du style expérimental ?». In Hollywood: le Temps des mutants. Pertuis : Rouge profond, 2013. 205-256.

---. « Knight of Cups : 'The child that I was' ». Positif 658 (2015) : 9-10.

Blasi, Gabriella. The Work of Terrence Malick: Time-Based Ecocinema. Amsterdam : Amsterdam University Press, 2020.

Bouvrande, Isabelle. «Phaos, lux et lumen : de la visibilité du monde à la visibilité de la peinture à la Renaissance ». In Lumière(s). Ed. Benoit Carré et al. Villeneuve d'Ascq : Publications de l'Institut de recherches historiques du Septentrion, 2016. $1^{\text {er }}$ février 2020. https://books.openedition.org/ irhis/601?lang=fr.

Bunyan, John. The Pilgrim's Progress. 1678. Oxford : Oxford University Press, 2003.

Cabau, Jacques. La prairie perdue : le roman américain. Paris : Seuil, 1966.

Calhoun, David H. «Entranced by the Spectacle of Truth: Wonder and Ascent in Plato and Terrence Malick's "Knight of Cups" and "To the Wonder" ». In Plato and the Moving Image. Ed. Shai Biderman and Michael Weinman. Leiden: Brill, 2019. 194-209. 
Caruana, John and Mark Cauchi (ed.). Immanent Frames: Postsecular Cinema between Malick and von Trier. New York: Suny Press, 2018.

Cavell, Stanley. The World Viewed: Reflections on the Ontology of Film. Cambridge : Harvard University Press, 1979 ; La Projection du monde : réflexions sur l'ontologie du cinéma. Trad. C. Fournier. Paris : Belin, 1999.

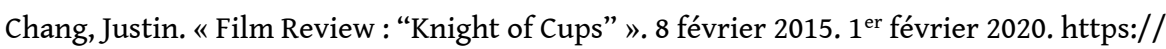
variety.com/2015/film/reviews/berlin-film-review-knight-of-cups-1201425546.

Chion, Michel. La Ligne rouge. Chatou : Éditions de la Transparence, 2005.

Ciment, Michel. « Projection privée » (avec Philippe Fraisse et Pierre Berthomieu). France culture. 28 novembre 2015. $1^{\text {er }}$ février 2020. http://www.franceculture.fr/emissions/projection-privee/ philippe-fraisse-et-pierre-berthomieu.

Dahan, Yaron. «Concrete Vanities: Terrence Malick's “Knight of Cups” ». mars 2015. $1^{\mathrm{er}}$ février 2020. http://sensesofcinema.com/2015/feature-articles/concrete-vanities-terrence-malicksknight-of-cups.

Dreyfus Hubert and Camilo Salazar Prince. « "The Thin Red Line:” Dying without Demise, Demise without Dying ». In The Thin Red Line. Ed. David Davies. New York: Routledge, 2009. 29-44.

Eliot, Thomas Stearns. The Wast Land and Other Poems. London: Faber and Faber, 1972.

Emerson, Ralph Waldo. Essays \& Lectures. New York : The Library of America, 1983.

Fraisse, Philippe. Un jardin parmi les flammes : le cinéma de Terrence Malick. Pertuis : Rouge profond, 2015.

Gaudeaux, Ariane. «La lumière dans les yeux des autres ». 14 décembre 2015. $1^{\mathrm{er}}$ février 2020. https://www.revue-eclipses.com/knight-of-cups/critique/-la-lumiere-dans-les-yeux-desautres-131.html.

Hadot, Jean. «Thomas Actes de ». Encyclopcedia Universalis. $1^{\text {er }}$ février 2020. https:// www.universalis.fr/encyclopedie/actes-de-thomas.

Hoffmeister Gerhart. " World as a Stage - "Theatrum Mundi” ». The Literary Encyclopedia. 23 mars

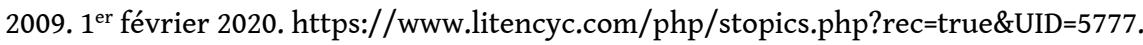

Ibsen, Henrik. Peer Gynt. 1867. Trad. F. Regnault. Paris : Gallimard, 2015.

Kierkegaard, Søren. Ou bien... ou bien. 1843. Trad. F. et O. Prior et M.-H. Guignot. Paris : Gallimard, 1984 [1].

---. Crainte et tremblement. 1843. Trad. P.-H. Tisseau. Paris : Aubier, 1984 [2].

---. Traité du désespoir. 1849. Trad. K. Ferlov et J.-J. Gateau. Paris : Gallimard, 1988.

---. Les stades immédiats de l'éros ou l'éros et la musique. 1843. Trad. P.-H. Tisseau et E.-M. Jacquet-

Tisseau. Paris : Le Bruit du temps, 2015.

Lacoue-Labarthe, Philippe. « Sublime ». 1991. Encyclopcedia Universalis. 2004.

Laughton, Charles. « The Story-Teller: A Session with Charles Laughton ». 1955. 19 novembre

2010. $1^{\text {er }}$ février 2020. https://archive.org/details/TheStory-teller.

Leigh, Jacob. « Unanswered Questions: Visions and Experience in Terrence Malick's “The Thin

Red Line" ». CineAction 62 (2003): 2-14.

Like Stories of Old. « "Knight of Cups:” Our Eternal Quest for Meaning-Kierkegaard's

Existentialism ». 2 juin 2017. $1^{\mathrm{er}}$ février 2020. https://ant.umn.edu/fijpchgsex/view. 
Logan, Trevor. « Kierkegaard in L. A.: Terrence Malick's “Knight of Cups” ». 14 mars 2016. $1^{\text {er }}$ février 2020. http://www.curatormagazine.com/trevor-logan/kierkegaard-in-l-a-terrencemalicks-knight-of-cups.

Malick, Terrence Frederick. «"The Tree of Life:" First Draft ». Writers Guild of America, 2007. $1^{\text {er }}$ février 2020. https://indiegroundfilms.files.wordpress.com/2014/01/tree-of-life-thejun-25-07-1st.pdf.

Martin, Adrian. « Things to Look Into: The Cinema of Terrence Malick ». Décembre 2006. $1^{\mathrm{er}}$ février 2020. http://www.rouge.com.au/10/malick.html.

Marx, Leo. The Machine in the Garden: Technology and the Pastoral Ideal in America. 1964. Oxford: Oxford University Press, 2000.

Mathis, Alexandre. «Knight of Cups de Terrence Malick : l'inconsolable solitude ». 27 novembre

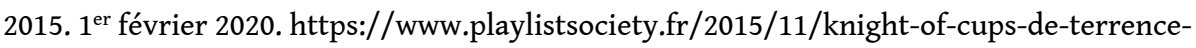
malick-linconsolable-solitude/123606.

Mattéi, Jean-François. « L'image du monde chez Stanley Cavell ou Celle qui n'était plus ». In Stanley Cavell : cinéma et philosophie. Ed. Sandra Laugier et Marc Cerisuelo. Paris : Presses de la Sorbonne Nouvelle, 2001. 21-36.

McCraken, Brett. «The Divine Guide in Terrence Malick's “Tree of Life” ». 21 mai 2012. $1^{\mathrm{er}}$ février 2020. https://www.brettmccracken.com/blog/2012/05/21/the-divine-guide-in-terrence-malickstree-of-life.

Ménard, Jacques-E. «Le 'Chant de la Perle' ». Revue des Sciences Religieuses 42 : 4 (1968) : 289-325. $1^{\mathrm{er}}$ février 2020. https://www.persee.fr/doc/rscir_0035-2217_1968_num_42_4_2516.

Nye, David E. American Technological Sublime. Cambridge: MIT Press, 1994.

Percy, Walker. The Moviegoer. 1961. New York: Vintage Books, 1998.

Platon. La République. Trad. G. Leroux. Paris : Flammarion, 2004.

---. Phèdre. Trad. L. Brisson. Paris : Flammarion, 2012.

Scott, A. O. « Review: In “Knight of Cups," a Writer's Flech Is Willing but His Spirit Is Weak ». 3

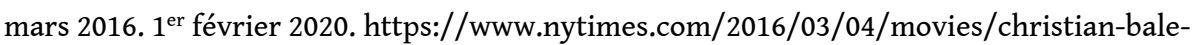
knight-of-cups-review.html.

Seaman, Natasha. « Artists of the Dark: "Night Visions: Nocturnes in American Art, 1860-1960" ".

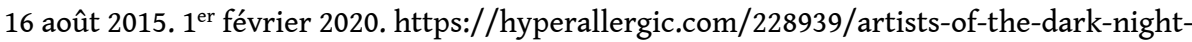
visions-nocturnes-in-american-art-1860-1960.

Sinnerbrink, Robert. Terrence Malick: Filmmaker and Philosopher. New York: Bloomsbury, 2019.

Smith, Henry Nash. Virgin Land: The American West as Symbol and Myth. 1950. Cambridge: Harvard University Press, 1970.

Solomon, Emily. « Interview: The Producers of "Knight of Cups" Including Emerson Alumni Sarah

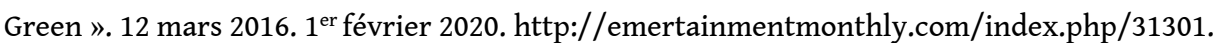

Sohrawardi, Shihab al-Din. « Le récit de l'exil occidental ». Trad. H. Corbin. L'Archange empourpré. Paris : Fayard, 1976. $1^{\text {er }}$ février 2020. http://www.archipress.org/batin/exil.htm.

Sragow, Michael. «Studies in Light and Place: the Production Designer Jack Fisk on Catching Life

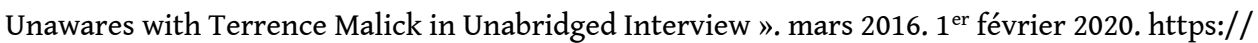
www.filmcomment.com/article/interview-jack-fisk. 
Strickland, Carol. « Shedding New Light on the Night: Obscurity and Clarity in the American

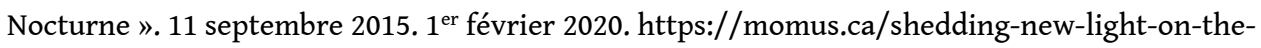
night-obscurity-and-clarity-in-the-american-nocturne.

Valance, Hélène. Nuits américaines : l'art du nocturne aux États-Unis, 1890-1917. Paris : Presses de l'université Paris-Sorbonne, 2015.

Whitney, E. Oliver. « Emmanuel Lubezki on "The Revenant," "Knight of Cups" and the

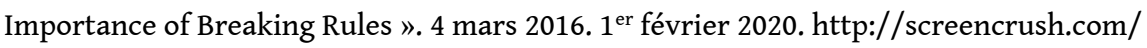
emmanuel-lubezki-interview.

Wittgenstein, Ludwig. Recherches philosophiques. 1956. Trad. F. Dastur et al. Paris : Gallimard, 2004. Wordsworth, William and Samuel Taylor Coleridge. Lyrical Ballads: 1798 and 1802. Oxford: Oxford University Press, 2013.

Young, Edward. The Complaint: or, Night Thoughts. 1742-1745. $1^{\mathrm{er}}$ février 2020. https:// www.gutenberg.org/files/33156/33156-h/33156-h.htm.

\section{NOTES}

1. Je remercie vivement les rapporteurs anonymes de la revue Miranda et Emmanuelle DelanoëBrun.

2. Le poème nous est parvenu par les Actes de Thomas, un texte chrétien apocryphe rédigé en syriaque au $3^{\text {ème }}$ siècle. Pour une traduction et un commentaire du poème, voir Jacques-E. Ménard, respectivement 298-308 et 309-325. Concernant les Actes, voir la notice de Jean Hadot dans Encyclopcedia Universalis. https://www.universalis.fr/encyclopedie/actes-de-thomas.

3. Les producteurs du film semblent renvoyer concernant cette fable, plutôt qu'au Chant de la Perle, au texte du philosophe et mystique perse Shihab al-Din Sohrawardi «Récit de l'exil occidental» (Solomon 2016). Le poème syriaque puise dans l'Apocalyptique juive et le christianisme, là où ce second texte intègre la tradition islamique tout en conservant des liens avec la tradition judéo-chrétienne et le platonisme, lesquels pourraient expliquer ses analogies évidentes avec le Chant de la Perle. Si le récit symbolique de Sohrawardi s'avère cohérent avec Knight of Cups, la fable évoquée par le père renvoie indéniablement au poème syriaque. Pour une discussion sur ce point, voir M. Gail Hamner dans Christopher B. Barnett et Clark J. Elliston 2016, 251, 270, et Gabriella Blasi 2020, 139-141. Pour le « Récit de l'exil occidental », voir la traduction qu'en a donnée Henry Corbin (Sohrawardi 1976).

4. On sait que Malick demanda à son interprète principal, Christian Bale, de lire le livre en amont du tournage.

5. "...the specific character of despair is precisely this: it is unaware of being despair. » La citation, non paginée, figure en exergue au livre.

6. Malick lit abondamment Kierkegaard pendant son premier cycle d'études philosophiques à Harvard dans les années soixante. En 1966, il obtient une «Rhodes Scholarship » et entame une thèse de second cycle à Oxford sur le concept de "monde » chez Heidegger, Kierkegaard et Wittgenstein. Comme l'ont noté certains commentateurs, les films contemporains de Malick résonnent de la philosophie de Kierkegaard. Concernant Knight of Cups, voir Robert Sinnerbrink 2019, 161-206, Trevor Logan 2016 et « Like Stories of Old » 2017.

7. Cette figure est évoquée dans Crainte et tremblement, 1984. Pour des rapprochements entre le chevalier de la foi et le soldat Witt dans La Ligne rouge (The Thin Red Line, 1998), voir Hubert Dreyfus et Camilo Salazar Prince 2009, 42 et Paul Martens dans Christopher B. Barnett et Clark J. Elliston 2016, 165. 
8. Celui-ci est issu d'une carte du tarot de Marseille, qui s'inspire du mythe du saint Graal.

9. Opéra de Ralph Vaughan Williams créé au Covent Garden de Londres en 1951, d'après le roman éponyme de Bunyan. La composition est interprétée par le City of London Sinfonia.

10. Pour des analogies avec le texte kierkegaardien, voir Logan 2016.

11. C'est grâce à l'inspiration orientale et au moment nocturne que nombre de peintres et photographes américains du début $\mathrm{du} 20^{\mathrm{ème}}$ siècle trouvèrent le moyen de se réconcilier avec les grandes métropoles de l'Est des États-Unis. Voir à ce sujet Hélène Valance 2015, en particulier 25. 12. L'enregistrement est issu de la série "The Story-Teller: A Session with Charles Laughton » (1955). 19 novembre 2010. $1^{\mathrm{er}}$ février 2020. https://archive.org/details/TheStory-teller.

13. Écrit en 1989 d'après le Psaume 51 et révisé en 1992, le morceau est interprété par le chœur de chambre philharmonique estonien.

14. Cette idée, qui constitue peut-être la pierre de touche de la métaphysique de Malick, est suggérée par Jacob Leigh au sujet de l'ouverture de La Ligne rouge (Leigh 5).

15. C'est entre deux chérubins que Moïse entend l'Éternel s'adresser à lui du haut du propitiatoire (Exode 25:2; Nombre 7:89). L'interprète de Rick, Christian Bale, ne porte pas seulement le même prénom que le héros de Bunyan. Il tourne aussi, à peu près au même moment que le film de Malick, Exodus (2014) de Ridley Scott, où il joue le rôle de Moïse.

16. Dans la tradition anglophone, un grand exemple est les Night Thoughts (1742-1745) d'Edward Young. On notera que Knight of Cups est divisé en neuf chapitres (le "soleil », signalé par une carte de tarot qui pivote dans une piscine plutôt que par un intertitre, étant unanimement ignoré de la critique), le nombre de « nuits » que compte le recueil de Young.

17. Voir sur ce sujet le quatrième chapitre de l'ouvrage de Hélène Valance, en particulier p. 109-110.

18. Les déambulations répétées de Rick dans ces décors rappellent la fin du Dernier Nabab (The Last Tycoon, 1976) d'Elia Kazan, où Monroe Stahr (Robert De Niro) disparaît dans les « backlots » de la Paramount. Si Knight of Cups multiplie les ponts avec ce dernier film (le séisme inaugural qui réveille le héros à lui-même en est, comme l'a noté Pierre Berthomieu [2015 10], un autre exemple), c'est sans doute parce que Malick, par-delà son admiration pour le cinéaste grec et leurs similitudes biographiques, a souhaité se réapproprier la forme du récit du Dernier Nabab, où le contexte hollywoodien constitue déjà une métaphore de la crise spirituelle du protagoniste.

19. Interprétée par le BBC Scottish Symphony Orchestra, la composition est légèrement remaniée par le cinéaste. Ce dernier en ramène l'andante original à un tempo plus lent, proche de l'adagio, qui accentue le caractère dramatique de la composition.

20. Publié en 1867, joué pour la première fois en 1876 au théâtre Christiania d'Oslo.

21. D'après le conte populaire recueilli par Peter Christen Asbjørnsen en 1843.

22. Issu du poème de T. S. Eliot, La Terre vaine (The Waste Land, 1922), le terme désigne par extension l'Amérique postérieure à l'anéantissement des mythes de la «terre vierge » et de la pastorale par le progrès technologique au tournant du $20^{\text {ème }}$ siècle. Bien que ces mythes fondateurs persistent sous certaines formes, le symbole dominant du pays devient la Metropolis. Sur la mythologie nationale, voir Henry Nash Smith (1970) et Leo Marx (2000). Sur la Metropolis dans la tradition américaine, voir Jacques Cabau (1981).

23. Pour une synthèse éclairante du postulat ontologique de l'ouvrage, voir Jean-François Mattéi (2001).

24. La phrase résonne du lexique du Voyage du Pèlerin et de l'arrivée du Prince en Égypte dans le Chant de la Perle («Et, parce que j'étais seul, que j’étais isolé, je fus un étranger pour les gens de ma demeure » [Ménard 304]). On mentionnera également la phrase de Binx Bolling au début de The Moviegoer, au sujet d'un héros de cinéma amnésique dont l'histoire ressemble à la sienne : "He found himself a stranger in a strange city " (Percy 4). Malick cite en l'occurrence l'épître de saint Paul aux Hébreux, que l'on rendra dans la traduction du roi Jacques : «they were strangers and pilgrims on the earth » (11:13). 
25. Les citations bibliques ci-après sont de la Bible Segond.

26. 1926, huile sur toile, 121,9 x 76,2 cm, Georgia O'Keeffe Museum, Santa Fe, NM. Voir la fiche du Minneapolis Institute of Art (Mia). https://collections.artsmia.org/art/2725/city-night-georgiaokeeffe.

27. Sur ces notions issues de la philosophie d'Aristote et de la scolastique, voir Isabelle Bouvrande 2016. Pour un exemple de leur application au cinéma, on se reportera à Jacques Aumont 2010.

28. Si l'existence moderne comme fragments est un thème typique du modernisme, les termes du père pourraient renvoyer à la philosophie d'Emerson, dans « The American Scholar » (« Le savant américain ", 1837) : " The state of society is one in which the members have suffered amputation from the trunk and strut about so many walking monsters,-a good finger, a neck, a stomach, an elbow, but never a man. Man is thus metamorphosed into a thing, into many things. » (Emerson 54) On rapprochera cette citation d'un passage du scénario de The Tree of Life au sujet de la vie moderne : "One is without form and void. Thousand-minded. At odds with himself. A miscellany, not a man. » (Malick 2007, 11)

29. C'est la formule de Wittgenstein pour exprimer le fait que le langage est la manière humaine d'être au monde (2004). La parataxe comme élément caractéristique du cinéma de Malick est une idée de Michel Chion (2005). Sur le même sujet, voir Adrian Martin (2006).

30. Cette terminologie biblique (Gn, 11:1-9) est reprise par Malick dans un passage urbain du scénario de The Tree of Life : «The sights of a modern city: it could be Chicago, New York, Houston, Paris, Mumbai, Los Angeles, or a composite of them all. We never see it whole-no skyline or defining monumentfragments only-a frenzy of things and people on the move-a continuous flow of trains and cars-a new Babel.» (2007 9)

31. Concernant cette notion romantique héritée du Pseudo-Longin, voir Philippe LacoueLabarthe 2004. Pour le «sublime électrique " lié à l'émergence de la métropole américaine, voir David Nye 1994. Pour sa place dans le nocturne américain, voir Hélène Valance 2015.

32. Série télévisée de quarante-sept épisodes et trois saisons réalisés par Mark Frost et David Lynch entre 1990 et 2017.

33. La première règle de ce dogme étant que toutes les autres peuvent être rompues à tout moment. Voir Benjamin B. 2011.

34. Interprétée par une actrice indienne, la jeune femme exécute, aux mélismes orientalisants de l'« épigraphe antique » Pour l'Égyptienne (1914) de Claude Debussy, des gestes du yoga dans un temple bouddhiste.

35. Dans le drame d'Ibsen, Solveig est la femme aimante et sincère que le protagoniste manque toute sa vie de reconnaître comme celle qu'il aime.

36. Comme la nomme un intertitre du film, d'après un arcane majeur du tarot de Marseille.

37. Notion baroque associée à l'œuvre de William Shakespeare, le "Théâtre du monde » trouverait son origine la plus ancienne dans la philosophie grecque, notamment avec l'allégorie de la caverne de Platon. Voir à ce sujet Gerhart Hoffmeister, «World as a Stage - "Theatrum

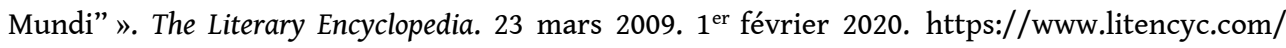
php/stopics.php?rec=true\&UID=5777.

38. On notera la proximité, davantage encore d'avec le « Donjon du doute » qu'évoque l'opéra de William, avec la «Foire aux vanités » (Vanity Fair) que traversent Christian et son compagnon Fidèle.

39. Vers 220-185 avant notre ère, $5,57 \mathrm{~m}$, sculpture en marbre, musée du Louvre, Paris.

40. L'enregistrement est issu de la même série citée plus haut (Laughton 2010).

41. Le film exploite ici une analogie extrêmement subtile avec Le Voyage du Pèlerin. Lorsque, à la tombée de la nuit, le héros de Bunyan traverse seul la Vallée de l'Ombre de la Mort, une voix dans l'obscurité lit le verset 4 du Psaume 23, sur le thème de l'Éternel comme protecteur dans l'adversité : «Quand je marche dans la vallée de l'ombre de la mort, / je ne crains aucun mal, car 
tu es avec moi : / ta houlette et ton bâton me rassurent. » Malick confère une forme proprement filmique à l'idée de Bunyan.

42. Vers 1513-16, huile sur bois, 69 × $57 \mathrm{~cm}$, musée du Louvre, Paris.

43. On verra dans cette représentation de la nuit urbaine un équivalent américain et ultramoderne aux nocturnes de James Abbot McNeill Whistler et d'Atkinson Grimshaw, dont le trait abstrait visait déjà à entremêler espaces urbain et naturel. Voir sur le sujet l'ouvrage de Hélène Valance (2015).

44. 1903 , photographie, $41,9 \times 35,3 \mathrm{~cm}$.

45. Dans la Bible, l'Esprit de Dieu est un « souffle » (rûah en hébreux, "vent », pneûma dans les évangiles rédigés en grec, spiritus en latin) qui circule parmi les éléments, l'eau (Gn, 2:2) ou, de façon privilégiée, le ciel (par exemple Lc, 3:22). On verra que dans Knight of Cups, ce souffle prend la forme d'une femme. Pour des parallèles, à mitiger, entre le cinéma de Malick et l'esprit biblique, voir la première partie de l'article de Brett McCraken 2012, et Chistopher B. Barnett dans le livre coédité avec Clark J. Elliston 2016, 103-107.

46. Ainsi que le note Pierre Berthomieu, cette figure rappelle la jeune femme guidant Jack dans le désert de The Tree of Life, nommée « Guide » au générique du film (Berthomieu 2013, 225 ; Ciment 2015). Elle fournit un pendant de chair aux statues d'anges filmées à Las Vegas (la reproduction de la Victoire de Samothrace, qui ne possède pas de tête, et celle de l'archange Gabriel, montrée de dos). Si cette femme-guide semble chez Malick l'incarnation symbolique du Saint-Esprit, sa présence à l'heure nocturne résonne de la tradition romantique. On citera, en littérature, le poème de Samuel Taylor Coleridge, «The Nightingale: A Conversation Poem » (publié dans les Lyrical Ballads rédigées avec William Wordsworth en 1798), où une jeune servante promène son enfant dans les bosquets nocturnes pour que le chant du rossignol dissipe ses mauvais rêves et le remplisse de joie. Ses mots à elle concluent le poème : "if that Heaven / Should give me life, his childhood shall grow up, / Familiar with these songs, that with the night / He may associate Joy! » (v. 106-109). Une telle figure se retrouve encore dans le nocturne américain, notamment avec les femmes musiciennes de Thomas Wilmer Dewing. Hélène Valance note que ces femmes associées à la nuit suggèrent « une certaine transcendance, le dépassement du simple visuel » (111).

47. D'après Socrate, dans le Phèdre, les cigales chantent pour indiquer à la déesse Calliope ceux des hommes « qui passent leur vie à aspirer à la sagesse » (2004 139).

48. Le guide cite le verset 12 du Psaume 139, adressé par David à l'Éternel : « même les ténèbres ne sont pas obscures pour toi, / la nuit brille comme le jour, / et les ténèbres comme la lumière ». Si cette jeune femme n'est pas le Saint-Esprit incarné, elle a indéniablement lu la Bible.

49. On a donc, en l'occurrence, une puissante inversion de l'allégorie platonicienne. Voir la République, livre VII, 514a-517a (2004 358-362).

\section{RÉSUMÉS}

Knight of Cups (2015) fait partie d'une trilogie contemporaine et expérimentale composée avec À la merveille (To the Wonder, 2012) et Song to Song (2017). À sa sortie, le film paraît déstabiliser le public et la critique en raison de son humeur sinueuse et mélancolique. On souhaite reconsidérer ces réactions en mettant au jour un motif: la «nuit». Puisant dans les traditions platonicochrétienne et romantique, Terrence Malick semble reprendre ce motif pour porter à l'écran une réflexion sur le désespoir et la fragmentation de la vie moderne. Tout en indiquant les 
principales sources d'inspiration du film, on s'attache à faire ressortir sa recherche esthétique et son originalité.

Knight of Cups (2015) is part of a contemporary and experimental trilogy made up with To the Wonder (2012) and Song to Song (2017). On its release, the film seems to put off both the audience and reviewers by its meandering moodiness. This article wishes to reconsider such responses by shedding light on a particular motif: the "night". Drawing from a Platonic, Christian and Romantic tradition, Terrence Malick seems to take up this motif to bring to the screen a reflection on despair and modern life. While indicating the film's main sources of inspiration, this article aims to highlight its aesthetical research and originality.

\section{INDEX}

Mots-clés : nuit, désespoir, amour, romantisme

Keywords : night, despair, love, romanticism

\section{AUTEURS}

\section{GUILAIN CHAUSSARD}

Doctorant en études cinématographiques

Université Paris-Est, LISAA

guilain.chaussard@gmail.com 